\title{
Enterprise Architecture Cybernetics for Global Mining Projects: Reducing the Structural Complexity of Global Mining supply Networks via Virtual Brokerage
}

\author{
Hadi Kandjani, Lian Wen, Peter Bernus \\ Centre for Enterprise Architecture Research and Management (CEARM) \\ School of ICT, Griffith University, Brisbane, Australia \\ \{H.Kandjani, L.Wen, P.Bernus\}@griffith.edu.au
}

\begin{abstract}
Keywords: Enterprise Architecture Cybernetics, Global Mining Projects, Global Mining Supply Networks, Structural complexity, Virtual Brokerage, Extended Axiomatic Design Theory
\end{abstract}

\begin{abstract}
This paper applies the emerging paradigm of Collaborative Networks (CNs) to the global mining supply chains (introducing the new concept of 'global mining supply networks') and demonstrates that the efficiency of the global mining projects initiated and managed by the global mining supply networks can be limited by the complexity of the supply network itself. This paper then presents methods and theoretical examples to calculate and reduce the structural complexity of the global mining supply networks and the complexity of creation of the global mining projects through applying the virtual brokerages to the global mining supply networks.
\end{abstract}

\section{INTRODUCTION}

The significance of creating collaborative networks (CNs) and maintaining them has dramatically increased for participant enterprises due to imperatives such as globalisation and the need for specialisation to be able to maintain excellence [1].

Also creating and maintaining CNs which further create dynamic responses to market opportunities (by creating virtual organizations) emerged as a new paradigm for doing business [2]. The main objective of a $\mathrm{CN}$ is to be able to conceive, create, configure (or re-configure), maintain and operate Virtual Organisations (VOs) for service and manufacturing and to do so dynamically, effectively and efficiently in terms of time and cost, while meeting high quality standards [3].

CNs can be thought of as an evolution of supply chain integration made possible partly by the world-wide removal of numerous economic and trade obstacles and partly by the opportunities that pervasive information and communication technologies create. The opportunities are appealing because they promise the ability for enterprises to create rich and timely responses to the variety of demands required by the environment, whereupon none of the members have the ability to do so; in Ashby's terminology the $\mathrm{CN}$ is able to create a requisite variety to manage the complexity and maintain a dynamic equilibrium with its environment [4].

Kandjani and Bernus proposed the use of Enterprise Engineering (EE) methods (e.g. GERAM [5], [6], [7]) based on Extended Axiomatic Design Theory [8], [9], [10] to limit the complexity of VOs - and of the CN itself [3]. This paper formulates methods to calculate and reduce the structural complexity of the global mining supply networks (as CNs) and the complexity of creation of the global mining projects (as VOs) through virtual brokerages.

Virtual brokerages are VOs themselves (i.e. they can be created, operated, maintained and decommissioned) making the $\mathrm{CN}$ an evolving entity from its management point of view.

The various connections and collaborations and cooperations among participant enterprises in a CN (with the associated processes, structures and information channels) may be built by partners themselves, or with the involvement of various (specialised) hubs. For example, global mining supply networks may set up brokerages that create global mining projects in order to have the requisite variety to deal with the complexity of the environment. However, other hubs may be built 
as well; e.g. Milanovic, Stefanovic et al. [11] define Aggregator Hubs, Broker Hubs, Collaboration Hubs and Translator Hubs, and each of these may exist on various levels of the Supply-Chain Operations Reference-model (SCOR) - such as Scope, Configuration and Business Activity [12]. However, to ensure the desired qualities (dynamic, effective, efficient ...) in responding to the demands of the environment, the complexity of the global mining supply network itself can be a problem and a main hindrance.

Heuristic methods and trial and error in the creation of global mining projects give rise to time delays, unanticipated costs and unsatisfactory quality of the service or of the manufactured product. A global mining supply network member may have several connections to several other potential partners, but in any given situation (such as at the time of dynamically planning a global mining project) the probability of being able to successfully use this connection is not 100 percent - and this introduces delays into the creation of the global mining project, thus in case of a complex manufacturing or service process the cost of these searches can escalate.

We believe that part of this problem can be eliminated if the structural complexity of the global mining supply network is monitored and adjusted. Therefore the main question of this article is: how to calculate and how to reduce the structural complexity of global mining supply networks?

Before proposing a method to reduce structural complexity, first it will be necessary to define a measure of structural complexity, and a way to calculate it. For this purpose the paper uses an upper boundary of Kolmogorov Complexity (KC) [13] to estimate the complexity of a global mining supply network. This paper also is based on the assumption that a large scale global mining supply network can be modelled as random network. This is a typical assumption for research in an abstract level. However, real large global mining supply networks are usually 'not' normal distributed random network.

In fact, most of the global mining supply networks are Power-law distributed scale-free networks. Therefore, some adjustments are required so the model can be used to give practical estimation. Those works are listed in the following research direction. The paper is structured as follows. Section 2.1 introduces Enterprise Architecture Cybernetics as a research framework and distinct field in the EA discipline in which to cast our proposed solution, while Section 2.2 discusses Kolmogorov Complexity as a formal measure to calculate the structural complexity of the global mining supply networks (and, in general, to measure the descriptional complexity of any type of object). Section 3 is the major contribution of this paper, which firstly introduces the application of Extended Axiomatic Design Theory to design global mining supply networks and global mining projects with sufficiently limited complexity. The importance of this is that as complexity grows, global mining project behaviours become increasingly harder to predict under all circumstances. Subsequently we demonstrate through two examples how virtual brokerage as a solution can contribute to satisfying design axioms by introducing Virtual Brokers in the global mining supply network in order to minimise the information content of the network and reduce the structural complexity of the global mining supply network, and the complexity of creating global mining projects.

\section{RESEARCH FRAMEWORK}

\subsection{Enterprise Architecture Cybernetics}

In the current paper we use the laws from systems thinking, information theory and complexity theory to demonstrate how to reduce the structural complexity of a global mining supply network, i.e. to reduce the amount of information necessary to describe (or model) the global mining supply network and of the creation of global mining projects. In fact we apply the laws and theories of EA Cybernetics [3] and demonstrate their application in reducing the structural complexity of global mining supply networks and creation of global mining projects via virtual brokerage.

The target audience for the proposed methods in this paper include researchers and executives in enterprises involved in global mining industry. As this work is at the theoretical foundation stage, 
more research and software based tools will be required for these methods so that it could be applied in practical situation to help an enterprise to optimize its architecture to maximize its ROI.

\subsection{Kolmogorov Complexity}

In this paper, we use Kolmogorov Complexity (KC) as a measure to define the complexity of a CN.

The concept of KC was developed by the Russian mathematician Andrey Kolmogorov [13]. KC is one of the key elements in information theory [14]; it provides a mathematical definition of the information quantity in individual objects, which can be abstracted as binary strings or integers. For about half a century, KC has been applied in various disciplines and became and important part of the theoretical foundations of computer science [15].

The KC, denoted as $K_{U}(x)$, of a string $x$ with respect to a universal computer $U$ is defined as the length $l$ of the shortest program $p$ running on $U$ that prints $x$ and halts. It is denoted as:

$$
K_{U}(x)=\min _{p: U(p)=x} l(p)
$$

If the computer already has some knowledge about $x$, for example the length of $x$ as $l(x)$, it may require a shorter program that prints $x$ and halt. In this case, we define the conditional KC as:

$$
K_{U}(x \mid l(x))=\min _{p: U(p, l(x))=x} l(p)
$$

Theorem 1 If $U$ is a universal computer, then $\forall V$ which is another universal computer, $\exists c$ (which is a constant), such that $\forall x \in\{0,1\}^{*}$ (i.e., for each binary number $x$ )

$$
K_{U}(x) \leq K_{V}(x)+c
$$

The proof can be found in [14] and will not be repeated here. Theorem 1 indicates the universality of KC; it shows that the difference of $\mathrm{KC}$ with respect to different computers is smaller than a constant. If the string $x$ is long, the difference of KC caused by different computers becomes trivial. Therefore, we can discuss $K C$ of a string $x$, denoted as $K(x)$ without referring to a particular computer.

In this paper, we use $\log n$ to mean $\log _{2} n$. We also define $\log ^{*} n=\log n+\log \log n+\log \log \log n+\ldots$ until the last positive term.

\section{APPLICATIONS OF ENTERPRISE ARCHITECTIURE CYBERNETICS IN GLOBAL MINING SUPPLY NETWORKS}

\subsection{Extended Axiomatic Design and Enterprise Engineering in Collaborative Networks}

Axiomatic Design Theory (ADT) [16]. is a theory of complex systems. According to ADT, complex systems are systems that can not be predicted for sure to always satisfy their functional requirements Therefore, ADT describes causes of emerging complexity, and proposes a formal design theory with two design axioms and techniques and methods that system designs must satisfy to minimise complexity. ADT also claims to codify in a discipline-independent way what a 'best design' is, and in particular aims at avoiding unnecessary complexity.

To be able to avoid unnecessary complexity of type (c) above, Kandjani and Bernus [8] extended ADT with the Recursion Axiom.

Axiom I: Independence Axiom [16]. 'The independence of Functional Requirements (FRs) must always be maintained.' (An $\mathrm{FR}_{\mathrm{i}}$ is independent of others if there exist 'design parameters' [DP] so that if changing one $\mathrm{FR}_{\mathrm{i}}$ only one $\mathrm{DP}_{\mathrm{i}}$ must change, whereupon [FR] = [[A]] * [DP]. Here [FR] is the vector of FRs, [DP] is the vector of DPs and [[A]] is the matrix mapping DPs to FRs. If [[A]] is diagonal then the design is uncoupled (full independence is achieved). If [[A]] is triangular then the 
design is decoupled (the implementation process is 'serializable'). Otherwise the design is coupled (the implementation process of DPs is not 'serializable').

Axiom II: Information Axiom [16]. 'Out of the designs that satisfy Axiom I that design is best which has the minimal information content.' (Suh defined information content (IC) as the negative logarithm of the probability that the system will always satisfy the functional requirements.)

Axioms I and II together intend to minimise the complexity of the system's architecture complexity type (b) and can be used to design less complex global mining supply networks and global mining projetcs. However, observe that complexity type (c) (complexity of the global mining supply networks and resulting global mining projects) is not automatically addressed by introducing AD into EE practice. Therefore, Kandjani and Bernus [8] recently proposed that Axioms I \& II must also be applied to the change system (the projects that create global mining projects / change the global mining supply network). This is the 'recursion' axiom (below), meaning that change projects (as a system of systems) not only must follow Axioms I \& II, but they themselves need to be axiomatically designed.

Axiom III: Recursion Axiom [8]: 'The system that designs a system must satisfy Axioms I and II of design.' Note: a system that satisfies Axioms I and II does not necessarily satisfy Axiom III. E.g. at a given moment in time in its life history a system may be considered moderately complex, but the same system may still be very hard to create or change.

Example 3.1: let us denote three consecutive stages of an evolving global mining supply network as $S_{1}, S_{2}$ and $S_{3}$. In stage $S_{1}$ the global mining supply network is operating and has a design that satisfies Axioms I \& II. Let $S_{2}$ be the stage of change, where $S_{2}$ is the original global mining supply network extended by a change project $P$. The task of $P$ is to create $S_{3}$. When the global mining supply network in $S_{1}$ creates $P$ it can mandate that $P$ must use Axioms I \& II to design $S_{3}$. However, $P$ itself may not satisfy Axioms I \& II. Thus, $P$ can be more complex than necessary and even though its mandate is to design $S_{3}$, the probability of success of this endeavour may be less than desired, i.e. $P$ does not satisfy Axioms I and II (even if it applies them to design $S_{3}$ ).

Axiom III states that $S_{1}$ not only has to mandate that $P$ use Axioms I \& II, but $S_{1}$ has to design $P$ using Axioms I \& II (in the interest of successful evolution). Therefore, among those design processes that apply Axioms I \& II to design a system, that process is best which itself satisfies axioms I \& II.

\subsection{Calculation of a Global Mining Supply Network’s Structural Complexity}

In the previous subsection, we introduced AD principles to reduce the complexity of global mining supply networks, global mining projects and their design process. In this subsection, we will use Kolmogorov Complexity (KC) as a formal measure to characterise the complexity of a global mining supply network.

A global mining supply network of $n$ participants with approximately $k$ connections can be modelled as a so-called random network $N$ with $n$ nodes and $k$ edges [17]. We define the structural complexity of the network as the KC of $N$, denoted as $K(N)$. An upper boundary of $K(N)$ can be estimated as follows ${ }^{1}$ :

$$
\begin{aligned}
K(N) & \leq K(N \mid n, k)+K(n)+K(k) \\
& \leq \log \left(\begin{array}{c}
n \times(n-1) / 2 \\
k
\end{array}\right)+\log ^{*} n+\log ^{*} k+c \\
& =\log \frac{\left(\left(n^{2}-n\right) / 2\right) !}{k ! \times\left(\left(n^{2}-n\right) / 2-k\right) !}+\log ^{*} n+\log ^{*} k+c
\end{aligned}
$$

\footnotetext{
${ }^{1}$ In this paper $k$-combination is represented as: $\left(\begin{array}{l}n \\ k\end{array}\right)=\frac{n !}{k !(n-k) !}$
} 
Applying Stirling's approximation $n ! \approx e^{-n} n^{n} \sqrt{2 n \pi}$, the formula above can be simplified as:

$$
\begin{aligned}
K(N) \leq & \frac{n(n-1)}{2} \log \frac{n(n-1)}{2}-\frac{n(n-1)-2 k}{2} \log \frac{n(n-1)-2 k}{2} \\
& -k \log k+\frac{1}{2} \log \frac{n(n-1)}{2 k(n(n-1)-k) \pi} \\
& +\log ^{*} n+\log ^{*} k+c
\end{aligned}
$$

Example 3.2: If we have a global mining supply network as a random network $N$ with 40 nodes and 400 edges, then the structural complexity of the network can be estimated as:

$$
\begin{aligned}
K(N) & \leq \frac{40 \times 39}{2} \log \frac{40 \times 39}{2}-\frac{40 \times 39-400}{2} \log \frac{40 \times 39-400}{2} \\
& -400 \log 400+\frac{1}{2} \log \frac{40 \times 39}{800(40 \times 39-400) \times 3.14} \\
& +\log * 40+\log * 400+c \\
& \approx 9,853+c \text { (bits) }
\end{aligned}
$$

\subsection{Reducing the Structural Complexity of Global Mining Supply Networks}

Subsection 3.2 uses $\mathrm{KC}$ as a formal measure to estimate the complexity of a global mining supply network. This subsection presents a method to create a hierarchy of (virtual) brokerage which cluster global mining supply network participants. The method can be mathematically shown to substantially reduce the complexity of global mining supply network. The virtual brokerage clusters the participants resulting in a self-organising / embedded distributed design architecture. Virtual brokerages are global mining projetcs themselves (VOs i.e. they can be created, operated, maintained and decommissioned) making the global mining supply network an evolving entity from its management point of view.

Kandjani and Bernus [8] argue that "(under certain circumstances) by distributing the design authority between system components on each level, it may be possible to limit the complexity of the system that any design authority needs to see." They propose that self-design results in minimised information content (IC) and thus creates a 'best design' from the point of view of Axiom II. The current paper applies and verifies this concept of self-design through calculations of complexity before and after introducing the virtual brokerage.

Virtual brokerage should therefore be designed and evolved by the global mining supply network and its stakeholders. In other words that global mining supply network-change initiative (global mining supply network change project / program) is most likely to succeed whose model used to create and control it is the simplest. This is because global mining supply network management (the designer of the global mining supply network's future) has to make decisions and plan using information about the change environment, and it is the global mining supply network participants themselves (forming virtual brokers) who have tacit knowledge therefore needing the least explicit information (thus simpler model) to predict/control the global mining supply network's change process to reduce the global mining supply network's structural complexity.

In other words, models used by these virtual brokers as 'embedded designers' can be simpler because they know the important distinctions between relevant states of the surrounding global mining supply network participants and environment. Conversely, virtual brokers can recognise two states as identical from the point of view of relevance to the change process, whereupon the same two states of the change environment would be considered different by 'outsiders' (external observers) who do not have the tacit knowledge of this environment. As a consequence, the requisite variety of the global mining supply network's change project / change program (Ashby, 1957; p202) [4] can be minimised through the change process being designed by virtual brokers. 
Then we use examples to demonstrate that introducing a suitable virtual brokerage in a global mining supply network can reduce the descriptional complexity of global mining supply network.

Let $N$ be a global mining supply network as a random network with $n$ nodes and $k$ edges. Then we introduce an extra node $V$ as a virtual brokerage. This node will work as a broker for $m$ nodes in $N$. Let us call the set of the $m$ nodes $M$. The new global mining supply network is called $N$ '.

As $N$ is a random network, the probability $p$ that any two nodes in network $N$ have a connection can be estimated as:

$$
p=2 k /\left(n^{2}-n\right)
$$

Then the possibility $p_{m}$ that for a node in $N-M$ originally has one or more connections with $M$ can be estimated as:

$$
p_{m}=1-(1-p)^{m}
$$

Therefore the number of connections between $N-M$ and $V$ can be estimated as:

$$
n_{v}=p_{m} \times(n-m)
$$

Then we can calculate the structural complexity of $N^{\prime}$ and eventually calculate how complexity decreased. An upper boundary of KC of $N$ ' can be estimated based as:

$K\left(N^{\prime}\right) \leq K(N-M)+K(m)+K(V) \approx K(N-M)+K(m)+\log \left(\begin{array}{c}n-m \\ n_{v}\end{array}\right)+c$

Following from Example 3.2, we create Example 3.3:

Example 3.3: Let $N$ be a global mining supply network with 40 nodes $(n=1000)$ and 400 edges $(k=400)$. If we introduce a new node $V$ which takes over the connection of 10 nodes $(m=10)$ from $N$, the new global mining supply network is denoted $N^{\prime}$, then the decrease of complexity $\Delta=K(N)-K\left(N^{\prime}\right)$ can be estimated as:

$p=2 k /\left(n^{2}-n\right)=0.5128$

$p_{m}=1-(1-p)^{m}=0.9992$

$n_{v}=p_{m} \times(n-m) \approx 31$

$K\left(N^{\prime}\right) \leq 5135+5.6+1+c<5142+c($ bits $)$

$\Delta=K(N)-K\left(N^{\prime}\right) \approx 9853-5142=4711$ (bits)

\section{CONCLUSIONS}

The article reviewed how the application of extended axiomatic design theory in enterprise engineering could limit the complexity of the global mining supply networks and resulting global mining projects. This article also demonstrated a method of reducing the structural complexity of global mining supply networks, thereby promoting the reduction of complexity of creation of global mining projects. The example provided also illustrates how the distribution of the global mining project design task among virtual brokers reduces the complexity that any global mining project creation authority needs to see. This is important not only for the initial creation stage of global mining projects, but also for the operational stage, in which efficient (agile) dynamic reconfiguration is often necessary [18].

\section{REFERENCES}

[1] M. Scherrer-Rathje, J. Arnoscht et al. A generic model to handle complexity in collaborative networks. In Proc. PICMET 2009. IEEE. (2009), pp271-287. 
[2] L. Camarinha-Matos and H. Afsarmanesh (Eds) Collaborative networked oganizations: a research agenda for emerging business models. London : Kluwer (2004).

[3] H. Kandjani and P. Bernus, Capability Maturity Model for Collaborative Networks based on Extended Axiomatic Design Theory. In L.M.Camarinha-Matos, A.A.Pereira Klen, H.Afsarmanesh (Eds.) Adaptation and Value Creating Collaborative NetworksProc. IFIP Advances in ICT Vol 362 Berlin : Springer. (2011a), pp421-427.

[4] W.R. Ashby, An introduction to cybernetics. Taylor \& Francis (1956).

[5] IFIP-IFAC-Task-Force, GERAM: Generalised enterprise reference architecture and methodology. V6.3 (1999).

[6] ISO15704, Industrial automation systems - Requirements for enterprise-reference architectures and methodologies. Geneva : ISO TC184.SC5.WG1 (2000, Amd.2005).

[7] P. Bernus and O. Noran (2010) A Metamodel for Enterprise Architecture. In Enterprise Architecture, Integration and Interoperability. IFIP Advances in ICT. Vol 326 Berlin : Springer. pp56-65.

[8] H. Kandjani and P. Bernus, Engineering Self-Designing Enterprises as Complex Systems Using Extended Axiomatic Design Theory. IFAC Papers On Line V18 (Part1) Amsterdam : Elsevier (2011b), pp11943-11948.

[9] N. Suh, Axiomatic Design: Advances and Applications. New York : Oxford Uni. Press (2001).

[10] N. Suh, Complexity: Theory and Applications. New York : Oxford University Press (2005).

[11] I. Milanovic, M. Stefanovic, et al. Supply Chain Information Integration Methods from the Quality Aspect. Proc. 5 ${ }^{\text {th }}$ Int. Quality Conf. University of Kragujevac : Faculty of Mechanical Eng. (2011) pp439-448.

[12] P. Bolstorff, and R. G. Rosenbaum Supply chain excellence: a handbook for dramatic improvement using the SCOR model. New York : Amacom Books (2007).

[13] A. N. Kolmogorov, On the Logical Foundations of Information Theory and Probability Theory. Problems of Information Transmission. 1(1) (1965), pp1-7.

[14] T.M. Cover and J.A. Thomas, Elements of information theory. New York : Wiley (2006).

[15] M. Li, and P. Vitányi, An introduction to Kolmogorov complexity and its applications. ( $3^{\text {rd }}$ Ed). Berlin : Springer (2008).

[16] N. Suh, The Principles of Design. New York : Oxford University Press (1990).

[17] B. Bollobás, Random graphs. (2 ${ }^{\text {nd }}$ Ed) New York : Cambridge Univ Press (2001).

[18] B. Clegg and M. Binder Managing the Dynamic Reconfiguration of Enterprises. In G. Putnik and M.M. Cruz-Cunha (Eds) Encyclopedia of networked and virtual organizations. Hershey : IGI Global (2008), pp882-890. 